\title{
NUMERICAL SOLUTION TO A SIDEWAYS PARABOLIC EQUATION*
}

\author{
Dinh Nho Hào ${ }^{\dagger}$ \\ Dipartimento di Matematica, Politecnico di Milano \\ Piazza Leonardo da Vinci, 32, 20133 Milano, Italy, \\ H.-J. Reinhardt, and A. Schneider \\ Universität-GH Siegen, FB Mathematik, Walter-Flex-Straße 3, 57068 Siegen, Germany
}

\begin{abstract}
The inverse heat conduction problem can be considered to be a sideways parabolic equation in the quarter plane. This is a model of a problem, where one wants to determine the temperature on both sides of a thick wall, but one side is inaccessible to measurements. A numerical procedure for this severely illposed problem is suggested, which consists in two steps namely a mollification of the data and a marching difference scheme. The numerical method is proved to be stable. Several computational results are presented and discussed.
\end{abstract}

\section{Introduction}

In many engineering contexts, it is sometimes necessary to estimate the surface temperature and the surface heat flux in a body from a measured temperature history at a fixed location inside the body. These so-called inverse heat conduction problems have been discussed by many authors (see, e.g., $[?, ?, ?, ?]$ and the references therein) and a number of solution methods has been proposed. In this paper we consider a model of such problems where one wants to determine the temperature on both sides of a thick wall, but one side is inaccessible to measurements. Such a situation is illustrated in Figure ??

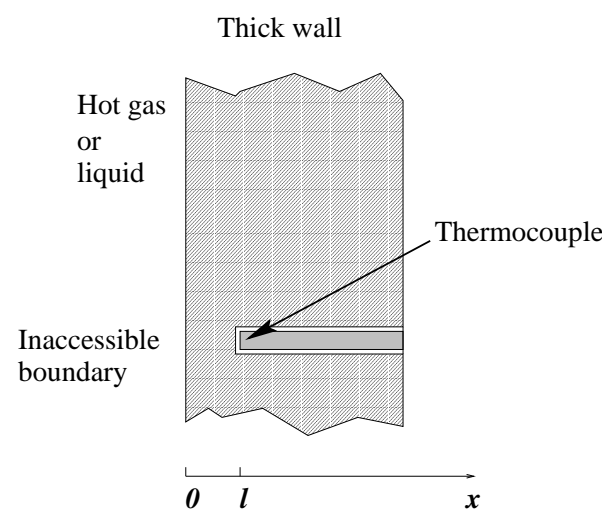

Figure 1: Determining surfaces temperatures from interior observations.

Mathematically, this problem can be formulated as an initial value problem for a parabolic equation in a quarter plane with data given along the line $x=l$. We refer to this problem as a sideways

\footnotetext{
*Part of this work has been taken from Schneider's dissertation.

${ }^{\dagger}$ On leave from Hanoi Institute of Mathematics supported by the CNR.
} 
parabolic equation. This problem is well known to be severely ill-posed, a small perturbation in the data may cause dramatically large errors in the solution (see the next section). Works have been done for such problems are mainly devoted for the heat equation, say, parabolic equations with constant coefficients (and we refer to this problem as the sideways heat equation), see, e.g., [?, ?, ?, ?, ?] and the references therein. Anderssen and Saull [?] proposed such a model of determining the surface history from bore hole measurements which leads to the above-mentioned problem and suggested a method for solving it. Manselli and Miller [?] obtained a stability estimate of Hölder type for the solution, they proposed the so-called mollification method for the solution. This idea is followed by many publications of Murio and his students (see [?] and the references therein). Levine [?], Levine and Vessella [?] obtained some similar estimates for the heat equation in multi-dimensional cases. Carasso [?] obtained stability estimates of Hölder type for the solution of the problem and proposed Tikhonov's regularization method and a marching difference scheme for solving the problem in a stable way. Carasso and his coworkers developed his idea further in other applications (for example, in testing gun barrels) as well as considered nonlinear models [?]-[?]. Talenti and Vessella [?] considered a similar problem with several data inside and obtained also a stability estimate of Hölder type. Eldén [?]-[?] proposed several methods as perturbation methods, finite difference methods and a method of lines. However, as said above, considering the problem these authors restricted themselves to the case of the heat equation, as they have an explicit integral representation for the solution. The latter is not available, if the coefficients of the parabolic equation describing the heat conduction process are not constant.

In [?] we considered a general case for a parabolic equation with variable coefficients. We gave there a stability estimate of Hölder type for the solution and indicated how to use the mollification method proposed by the first author [?, ?] for solving the problem numerically in a stable way. In fact, we shall first solve the well-posed "direct" problem to find the heat flux at the line where the data are given. As a result we get a non-characteristic where the theoretical aspects of the problem are studied, the emphasis of this work lies in the numerical approximation of the problem, the proof of the stability of the for a parabolic equation with noisy data which is severely ill-posed (see Figure ??). To solve this ill-posed problem we shall mollify the Cauchy data by the Dirichlet kernel in such a way that our problem becomes stable and the error estimate between the exact solution and the solution of the mollified problem is of Hölder type. To solve the mollified problem numerically we propose a very simple stable marching difference scheme for it and also prove the convergence of the scheme. Contrary to [?] where the theoretical aspects of the problem are studied, the emphasis of this work lies in the numerical approximation of the problem, the proof of the stability of the proposed marching scheme and the presentation of computational results.

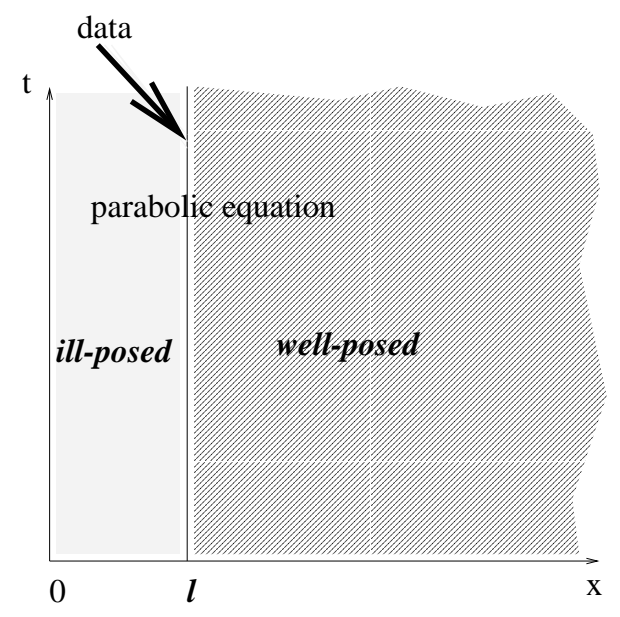

Figure 2: Splitting into a well-posed problem and an ill-posed Cauchy problem. 
In the next paragraph the mathematical model of our problem will be described. In paragraph ?? a stable marching difference scheme for solving our problem will be presented. Paragraph ?? aims to prove the stability results in paragraph ??. The reader who is not interested in this theoretical part should avoid it. The last paragraph is devoted to numerical examples.

\section{Problem setting}

The mathematical model of our problem can be described as the sideways parabolic equation in the quarter plane

$$
\begin{array}{cl}
u_{t}=a(x) u_{x x}+b(x) u_{x}+c(x) u, & x \in(0, \infty), \quad t \in(0, \infty), \\
u(l, t)=g(t), & t \in(0, \infty), \\
u(x, 0)=0, & x \in(0, \infty),
\end{array}
$$

We consider only bounded solutions of this problem. Here $l$ is a given positive number, $a, b, c$ are given functions such that for some $\lambda, \Lambda>0, B \geq 0$

$$
\lambda \leq a(x) \leq \Lambda, \quad|b| \leq B, \quad x \in(0, \infty),
$$

and

$$
c(x) \leq 0
$$

For simplicity, we suppose that

$$
a(\cdot) \in C^{2}(0, \infty), \quad b(\cdot) \in C^{1}(0, \infty), \quad c(\cdot) \in C(0, \infty) .
$$

Furthermore, throughout the paper, we suppose

$$
g \in L_{2}(0, \infty)
$$

and it is approximately given by $g_{\delta} \in L_{2}(0, \infty)$ :

$$
\left\|g-g_{\delta}\right\|_{L_{2}(0, \infty)} \leq \delta .
$$

The function $g_{\delta}$ is considered as the measured data in our problem.

The problem (??)-(??) is well known to be severely ill-posed: a small perturbation in the data $g$ may cause dramatically large errors in the solution $u(x, t)$ for $x \in[0, l)$. In fact, we have proved in [?] that the mappings from $u(0, t)$ into $u(l, t)$ and $u_{x}(l, t)$ are infinitely smoothing. It follows that our inverse problem is severely ill-posed. We give however here an explicit example of Talenti and Vessella [?] to emphasize this fact.

Consider the sideways heat equation

$$
\begin{array}{cl}
u_{t}=u_{x x}, & x \in(0, \infty), \quad t \in(0, \infty), \\
u(1, t)=g(t), & t \in(0, \infty), \\
u(x, 0)=0, & x \in(0, \infty) .
\end{array}
$$

We see that with

$$
g=g_{n}(t):=u_{n}(1, t)=\left(1+\frac{1}{n}\right) \frac{1}{t^{3 / 2}} \exp \left(-\frac{1}{4 t}\left(1+\frac{1}{n}\right)^{2}\right)
$$

there exists a unique solution

$$
u_{n}(x, t)=\left(x+\frac{1}{n}\right) \frac{1}{t^{3 / 2}} \exp \left(-\frac{1}{4 t}\left(x+\frac{1}{n}\right)^{2}\right) .
$$


Although

$$
\int_{0}^{\infty} u^{2}(1, t) d t=4\left(1+\frac{1}{n}\right)^{-2} \rightarrow 4
$$

we have

$$
\int_{0}^{\infty} u^{2}(0, t) d t=4 n^{2} \rightarrow \infty
$$

Thus our problem is ill-posed.

In [?] we proposed two similar ways to solve our problem numerically in a stable way. In this paper we shall follow only one of them. Namely, to do this we first solve the well-posed problem (see Figure ??)

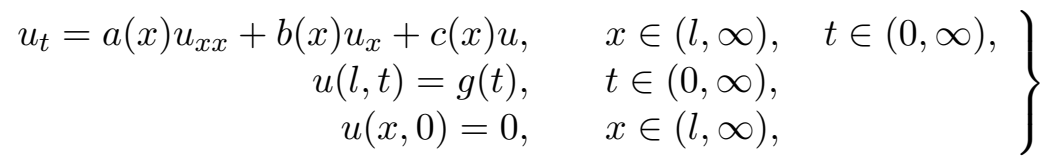

to find $h(t):=u_{x}(l, t)$. As a result we get

$$
\left.\begin{array}{rl}
u_{t}=a(x) u_{x x}+b(x) u_{x}+c(x) u & x \in(0, l), \quad t \in(0, \infty), \\
u(l, t)=g(t), & t \in(0, \infty), \\
u_{x}(l, t)=h(t), & t \in(0, \infty), \\
u(x, 0)=0, & x \in[0, l] .
\end{array}\right\}
$$

As $g$ is inexactly given, and $h$ can only be approximately found, the Cauchy-Problem (??) is severely ill-posed. Therefore we have to use a stable method for it. Namely, we shall use the the Dirichlet kernel to mollify the Cauchy data in such a way that (??) becomes well-posed within the mollification and the error estimate between the exact solution and the solution of the mollified problem is of Hölder type. To solve the mollified problem numerically we shall use a stable marching difference scheme for it. We note that our mollification method is different from that of [?].

\section{Stable marching difference scheme}

In this paragraph we describe the finite difference method for approximating the Cauchy data $u_{x}(l, t)$ with the inexact $g_{\delta}$ and then present the mollification method with a stable marching difference scheme for solving the Cauchy problem (??).

\subsection{Approximation to $u_{x}(l, t)$}

To approximate $u_{x}(l, t)$, we solve the well-posed problem (??) with the inexact data $g_{\delta}$. In doing so we first mollify $g_{\delta}$ by

$$
g_{\delta, \alpha}:=\frac{1}{\sqrt{2 \pi}} \int_{0}^{\infty} g_{\delta}(t) \frac{\sin \alpha(t-\tau)}{t-\tau} d \tau,
$$

where for $t<0$ the function $g_{\delta}$ is extended by 0 . The function $g_{\delta, \alpha}$ has some nice properties [?, pp. 316-318]: i) it belongs to $L_{2}(\mathbb{R})$, ii) it is an entire function of exponential type and its Fourier transform has compact support $[-\alpha, \alpha]$, iii) it is a good approximation to $g_{\delta}$ for sufficiently large $\alpha$. Since (??) is well-posed ([?]) we can choose $\alpha$ such that the solution of (??) with the new datum $g_{\delta, \alpha}$ is a good approximation to the $u(x, t)$ for $x \geq l$. For simplicity of notion we denote $g_{\delta, \alpha}$ by $G$ and the solution of (??) with these new data again by $u(x, t)$.

In practice, the solution of (??) shall be computed only for $0 \leq t \leq T$ for some $T>0$. The problem for $0 \leq t \leq T$ can be discretized by replacing the derivatives by difference quotients as follows. Consider the grid

$$
\left\{x_{n}=l+n h, t_{m}=m \tau: m=0,1, \ldots, M, \tau=T / M, n \geq 0\right\} .
$$


For a function $\mathcal{W}$ defined on $[l, \infty) \times[0, T]$, let

$$
\mathcal{W}_{m}^{n}:=\mathcal{W}\left(x_{n}, t_{m}\right)
$$

Then, (??) is discretized by

$$
\left.\begin{array}{rlrl}
a^{n} \frac{u_{m}^{n+1}-2 u_{m}^{n}+u_{m}^{n-1}}{h^{2}}+b^{n} \frac{u_{m}^{n+1}-u_{m}^{n-1}}{2 h}+c^{n} u_{m}^{n} & =\frac{u_{m+1}^{n}-u_{m}^{n}}{\tau}, & & 0 \leq m \leq M, n \in \mathbb{N}, \\
u_{m}^{0} & =G_{m}, & & 0 \leq m \leq M, \\
u_{0}^{n} & =0, & & n \geq 0,
\end{array}\right\}
$$

The solution of the scheme (??) is quite easily found: for $0 \leq m \leq M$ and $n \geq 0$

$$
\begin{aligned}
u_{m}^{0} & =G_{m}, \\
u_{0}^{n} & =0 \\
u_{m+1}^{n} & =\left(1+\tau\left(c^{n}-2 \frac{a^{n}}{h^{2}}\right)\right) u_{m}^{n}+\tau\left(\frac{a^{n}}{h^{2}}+\frac{b^{n}}{2 h}\right) u_{m}^{n+1}+\tau\left(\frac{a^{n}}{h^{2}}-\frac{b^{n}}{2 h}\right) u_{m}^{n-1} .
\end{aligned}
$$

In the next paragraph we shall prove the following result concerning the stability of the scheme (??).

Theorem 3.1 The finite difference scheme (??) approximates the problem (??) (for the data $G=$ $\left.g_{\delta, \alpha}\right)$ with a truncation error behaving like $O\left(h^{2}+\tau\right)$. If $h \leq \frac{2 \lambda}{B}$ and $\frac{\tau}{h^{2}}=\rho \leq \frac{1}{2 A}$, then it is conditionally stable and

$$
\sup _{0 \leq k \leq \infty}\left|u_{m}^{k}\right| \leq 2 \alpha^{3 / 2} T e^{c_{0} T}\|G\|_{2}, \quad 0 \leq m \leq M
$$

with $c_{0}$ being some definite constant.

\subsection{A stable marching difference scheme}

Assume now that the solution of the sideways parabolic equation (??)-(??) exists and at $x=0$, as a function of $t$, it belongs to $H^{s}(\mathbb{R})$ for some $s \in \mathbb{R}$, i.e. $f(t)=u(0, t)$ and $f \in H^{s}(\mathbb{R})$, where $u$ is the exact solution of (??) (for the notion of the standard Sobolev space $H^{s}$ we refer the reader to [?, ?]). Here and thereafter if we speak of a function of $t$ defined only for $t \geq 0$ for all $t \in \mathbb{R}$, we mean that this function is extended by 0 for $t<0$.

In the previous section we see that from the measured data $g_{\delta}$ we can have a good approximation to $u_{x}(l, t):=h(t)$. In fact, we can choose $\alpha$, and $h, \tau$ in (??) such that this approximation, denoted by $h_{\epsilon}$, satisfies

$$
\left\|h-h_{\epsilon}\right\|_{L_{2}(\mathbb{R})} \leq \epsilon,
$$

where $\epsilon$ is near $\delta(\epsilon \geq \delta)$.

Further, it is clear that

$$
\left\|g-g_{\delta}\right\|_{L_{2}(\mathbb{R})} \leq \epsilon .
$$

Thus, the where the theoretical aspects of the problem are studied, the emphasis of this work lies in the numerical approximation of the problem, the proof of the stability of the proposed marching scheme and the presentation of computational results. (??) has now two inexact Cauchy data $g_{\delta}$ and $h_{\epsilon}$. To solve it in a stable way, we first mollify the data $g_{\delta}$ and $h_{\epsilon}$ by convolutions with the Dirichlet kernel:

$$
g_{\delta, \nu}(t):=\int_{-\infty}^{\infty} g_{\delta}(t) \frac{\sin \nu(t-\tau)}{t-\tau} d \tau,
$$

and

$$
h_{\epsilon, \nu}(t):=\int_{-\infty}^{\infty} h_{\epsilon}(t) \frac{\sin \nu(t-\tau)}{t-\tau} d \tau,
$$


then, instead of solving (??) with inexact Cauchy data $g_{\delta}, h_{\epsilon}$, we consider its mollified version

$$
\left.\begin{array}{rl}
u_{\nu t}=a(x) u_{\nu x x}+b(x) u_{\nu x}+c(x) u_{\nu}, & x \in(0, l), \quad t \in(0, \infty), \\
u_{\nu}(l, t)=g_{\delta, \nu}(t), & t \in(0, \infty), \\
u_{\nu x}(l, t)=h_{\epsilon, \nu}(t), & t \in(0, \infty), \\
u_{\nu}(x, 0)=0, & x \in[0, l] .
\end{array}\right\}
$$

Take

$$
\nu=\nu^{*}:=2\left(\frac{1}{L} \ln \left(\frac{1}{\epsilon}\left(\ln \frac{1}{\epsilon}\right)^{-2 s}\right)\right)^{2} .
$$

With this choice of the mollification parameter our where the theoretical aspects of the problem are studied, the emphasis of this work lies in the numerical approximation of the problem, the proof of the stability of the proposed marching scheme and the presentation of computational results. is well-posed and an error estimate of Hölder type is obtained as Theorem ?? of the next paragraph will show.

To solve the mollified problem (??) numerically in a stable way, we shall propose for it a stable marching difference scheme. Again we consider a uniform grid on the $[0, l] \times \mathbb{R}$ plane:

$$
\left\{x_{n}=n h, t_{m}=m \tau: n=0,1, \ldots, N, h=l / N, m \in \mathbb{Z}\right\} .
$$

For a function $\mathcal{W}$ defined on $[0, l] \times \mathbb{R}$ set

$$
\mathcal{W}_{m}^{n}:=\mathcal{W}\left(x_{n}, t_{m}\right)
$$

Letting $U:=u_{\nu}, W:=U_{x}, \Psi(t):=g_{\delta}(t), \Phi(t):=h_{\varepsilon}(t)$, we conclude that (??) can be rewritten as

$$
\begin{aligned}
U_{x} & =W \quad, x \in(0, l), t \in \mathbb{R} \\
a(x) W_{x}+b(x) W+c(x) U & =U_{t}, x \in(0, l), t \in \mathbb{R} \\
U(l, t) & =\Psi(t), t \in \mathbb{R}, \\
W(l, t) & =\Phi(t), t \in \mathbb{R}, \\
U(x, 0) & =0 \quad, x \in[0, l] .
\end{aligned}
$$

As in [?], this problem is discretized by

$$
\left.\begin{array}{rlrl}
\frac{U_{m}^{n+1}-U_{m}^{n}}{h} & =W_{m}^{n+1} & & , n=0,1, \ldots, N, m \in \mathbb{N}, \\
a^{n} \frac{W_{m}^{n+1}-W_{m}^{n}}{h}+b^{n} W_{m}^{n}+c^{n} U_{m}^{n} & =\frac{U_{m+1}^{n}-U_{m-1}^{n}}{2 \tau}, & n=0,1, \ldots, N, m \in \mathbb{N}, \\
U_{m}^{N} & =\Psi_{m} & & m \in \mathbb{N} \\
W_{m}^{N} & =\Phi_{m} & & m \in \mathbb{N} \\
U_{0}^{n} & =0 & & n=0,1, \ldots, N .
\end{array}\right\}
$$

The unconditional stability of this difference scheme can be proved using the discrete Fourier transform in a way completely analogous to the argument in [?].

Theorem 3.2 The difference scheme (??) approximates the problem (??) with a truncation error behaving like $O\left(h+\tau^{2}\right)$. Furthermore, if $h$ is sufficiently small and $\tau \leq \pi / \nu$, then it is unconditionally stable and

$$
\max \left\{\left\|U^{n}\right\|_{l^{2}},\left\|W^{n}\right\|_{l^{2}}\right\} \leq e^{l(\lambda+B+C+\nu) / \lambda}\left(\|\Psi\|_{2}+\|\Phi\|_{2}\right) .
$$

\section{Proofs}

\subsection{Proof of Theorem ??}

Since in the scheme (??), $u_{m+1}^{n}$ is a linear combination of $u_{m}^{n-1}, u_{m}^{n}$ and $u_{m}^{n+1}$ (see also [?]), we have $u_{m}^{n}=0$ for $n>m$ and it follows

$$
\left|u_{m}^{n}\right| \rightarrow 0 \text { as } n \rightarrow \infty, 0 \leq m \leq M .
$$


Since $u_{x x}$ exists, the difference scheme (??) is consistent. Further, since $u$ is sufficiently smooth ([?]), (??) has a local truncation error behaving like $O\left(h^{2}+\tau\right)^{1}$. Now we will see that it is stable for an appropriate choice of $h$ and $\tau$. This fact is proved by the matrix method (see [?, ?]). The scheme (??) can be written as

$$
\begin{aligned}
u_{m+1}^{n} & =\left(1+\tau\left(c^{n}-2 \frac{a^{n}}{h^{2}}\right)\right) u_{m}^{n}+\tau\left(\frac{a^{n}}{h^{2}}+\frac{b^{n}}{2 h}\right) u_{m}^{n+1}+\tau\left(\frac{a^{n}}{h^{2}}-\frac{b^{n}}{2 h}\right) u_{m}^{n-1}, \\
u_{m}^{0} & =G_{m}, \\
u_{0}^{n} & =0,
\end{aligned}
$$

where $0 \leq m \leq M$ and $n \geq 0$. For $0 \leq m \leq M$ let $\{u\}_{m}$ be the vector $\left(u_{m}^{0}, u_{m}^{1}, u_{m}^{2}, \ldots\right)^{T}$ and $\{u\}_{m}^{*}=$ $\left(u_{m+1}^{0}-u_{m}^{0}, 0,0, \ldots\right)^{T}$ for $0 \leq m<M$. Setting $\rho:=\frac{\tau}{h^{2}}, w_{n}:=1+\rho\left(c^{n} h^{2}-2 a^{n}\right), y_{n}:=\rho\left(a^{n}+\frac{b^{n}}{2} h\right)$ and $z_{n}:=\rho\left(a^{n}-\frac{b^{n}}{2} h\right), n \geq 1$, the finite difference approximation (??) takes the form

$$
\{u\}_{m+1}=H\{u\}_{m}+\{u\}_{m}^{*}, 0 \leq m<M,
$$

where the matrix $H$ is given by

$$
H=\left(\begin{array}{cccccc}
1 & 0 & 0 & \ldots & & \\
z_{1} & w_{1} & y_{1} & 0 & \ldots & \\
0 & z_{2} & w_{2} & y_{2} & 0 & \ldots \\
0 & 0 & z_{3} & w_{3} & y_{3} & \\
\vdots & \vdots & & \ddots & \ddots & \ddots
\end{array}\right)
$$

For a vector $y$ and a matrix $G=\left(g_{n, k}\right)_{n, k \geq 0}$, let

$$
\begin{aligned}
\|y\|_{\infty} & :=\sup _{n \geq 0}\left|y_{n}\right| \\
\|G\|_{\infty} & :=\sup _{n \geq 0} \sum_{k \geq 0}\left|g_{n, k}\right| .
\end{aligned}
$$

It is clear that $\|G y\|_{\infty} \leq\|G\|_{\infty} \cdot\|y\|_{\infty}$. Hence

$$
\left\|\{u\}_{m+1}\right\|_{\infty} \leq\|H\|_{\infty} \cdot\left\|\{u\}_{m}\right\|_{\infty}+\left\|\{u\}_{m}^{*}\right\|_{\infty}, m \geq 0
$$

and one can see that if we would have

$$
\|H\|_{\infty} \leq 1+C_{0} \tau
$$

with $C_{0}$ being a positive constant, then

$$
\begin{aligned}
\left\|\{u\}_{m+1}\right\|_{\infty} & \leq\left(1+C_{0} \tau\right)\left\|\{u\}_{m}\right\|_{\infty}+\left\|\{u\}_{m}^{*}\right\|_{\infty} \\
& \vdots \\
& \leq\left(1+C_{0} \tau\right)^{m+1}\left\|\{u\}_{0}\right\|_{\infty}+\sum_{j=0}^{m}\left(1+C_{0} \tau\right)^{m-j}\left\|\{u\}_{j}^{*}\right\|_{\infty} \\
& \leq \sum_{j=0}^{m}\left(1+C_{0} \tau\right)^{T / \tau}\left|u_{j+1}^{0}-u_{j}^{0}\right| \leq e^{C_{0} \cdot T} \sum_{j=0}^{m}\left|G_{j+1}-G_{j}\right|,
\end{aligned}
$$

since $u_{0}^{n}=0, n \geq 0$. From the Bernstein-Nikolskii inequality for the entire function of exponential type $G[$ ?, p. 115], we have for $j \geq 0$

$$
\left|G_{j+1}-G_{j}\right|=\tau\left|\frac{G_{j+1}-G_{j}}{\tau}\right| \leq \tau\left\|G^{\prime}\right\|_{L_{\infty}(\mathbb{R})} \leq \tau \alpha\|G\|_{L_{\infty}(\mathbb{R})}
$$

\footnotetext{
${ }^{1}$ The boundary conditions $u(l, t)=G(t)$ and $\lim _{x \rightarrow \infty} u(x, t)=0$ and the initial condition $u(x, 0)=0$ are exact in our difference scheme.
} 
Further, according to the inequality of different metrics for entire functions of exponential type ([?, p. $126]):\|G\|_{L_{\infty}(\mathbb{R})} \leq 2 \alpha^{1 / 2}\|G\|_{L_{2}(\mathbb{R})}$, we get

$$
\left|G_{j+1}-G_{j}\right| \leq 2 \tau \alpha^{3 / 2}\|G\|_{L_{2}(\mathbb{R})} .
$$

Hence,

$$
\left\|\{u\}_{m+1}\right\|_{\infty} \leq 2 \alpha^{3 / 2} \tau(m+1) e^{C_{0} T}\|G\|_{L_{2}(\mathbb{R})}, 0 \leq m<M .
$$

Since $(m+1) \tau \leq M \tau=T$, this yields

$$
\left\|\{u\}_{m}\right\|_{\infty} \leq 2 \alpha^{3 / 2} T e^{C_{0} T}\|G\|_{2}, 0 \leq m \leq M .
$$

Thus, the difference scheme (??) is stable if $\|H\|_{\infty} \leq 1+C_{0} \tau$. We will see that this is the case for the right choice of $\tau$ and $h$. First, we have

$$
\|H\|_{\infty}=\max \left\{1, \sup _{n \geq 0}\left(\left|w_{n}\right|+\left|y_{n}\right|+\left|z_{n}\right|\right)\right\} .
$$

Using the properties of the coefficients $a, b, c$ (see (??) and (??)), for

$$
h \leq \frac{2 \lambda}{B}
$$

it follows that

$$
\left|\frac{b^{n}}{2 a^{n}} h\right| \leq 1
$$

and for

$$
\rho \leq \frac{1}{2 \Lambda}
$$

we have

$$
\left|w_{n}\right| \leq\left|1-2 \rho a^{n}\right|+\left|c^{n} \tau\right|=1-2 \rho a^{n}+\left|c^{n} \tau\right| .
$$

Hence

$$
\begin{aligned}
& y_{n}=\rho\left(a^{n}+\frac{b^{n}}{2} h\right)=\rho a^{n}\left(1+\frac{b^{n}}{2 a^{n}} h\right) \geq 0, \\
& z_{n}=\rho\left(a^{n}-\frac{b^{n}}{2} h\right)=\rho a^{n}\left(1-\frac{b^{n}}{2 a^{n}} h\right) \geq 0,
\end{aligned}
$$

and we conclude

$$
\begin{aligned}
\left|w_{n}\right|+\left|y_{n}\right|+\left|z_{n}\right| & \leq 1-2 \rho a^{n}+\left|c^{n} \tau\right|+y_{n}+z_{n} \\
& =1-2 \rho a^{n}+\left|c^{n} \tau\right|+\rho\left(a^{n}+\frac{b^{n}}{2} h\right)+\rho\left(a^{n}-\frac{b^{n}}{2} h\right) \\
& =1+\left|c^{n} \tau\right| \leq 1+C \tau, n \geq 0 .
\end{aligned}
$$

This yields $\|H\|_{\infty} \leq 1+C \tau$. Furthermore, for any $\varepsilon>0$ we find a number $N_{\varepsilon} \in \mathbb{N}$ such that

$$
\left|u_{m}^{n}-u\left(x_{n}, t_{m}\right)\right|=\left|u\left(x_{n}, t_{m}\right)\right|<\varepsilon, n \geq N_{\varepsilon}
$$

and thus we have proved Theorem ??

Remark 4.1 It is also possible to prove the conditional stability of (??) for the spectral norm

$$
\|H\|_{S}:=\sqrt{\rho\left(H^{*} H\right)}
$$

where $\rho(\cdot)$ is the spectral radius, and $H^{*}=\bar{H}^{T}$ is the associated conjugate transpose. Hence, one can also prove that (??) is conditionally stable with respect to the $l^{2}-$ norm.

Remark 4.2 As $G$ is an entire function of exponential type, the conditional stability of the problem (??) can be proved via the discrete Fourier transform technique. 


\subsection{Error estimate of the mollification method for the Cauchy problem}

Since we can split (??) into two independent where the theoretical aspects of the problem are studied, the emphasis of this work lies in the numerical approximation of the problem, the proof of the stability of the proposed marching scheme and the presentation of computational results. $s([?])$ :

$$
\left.\begin{array}{rl}
u_{t}^{1}=a(x) u_{x x}^{1}+b(x) u_{x}^{1}+c(x) u^{1}, & x \in(0, l), \quad t \in(0, \infty), \\
u^{1}(l, t)=g(t), & t \in(0, \infty), \\
u_{x}^{1}(l, t)=0, & t \in(0, \infty), \\
u^{1}(x, 0)=0, & x \in[0, l]
\end{array}\right\}
$$

and

$$
\left.\begin{array}{rl}
u_{t}^{2}=a(x) u_{x x}^{2}+b(x) u_{x}^{2}+c(x) u^{2}, & x \in(0, l), \quad t \in(0, \infty), \\
u^{2}(l, t)=0, & t \in(0, \infty), \\
u_{x}^{2}(l, t)=h(t), & t \in(0, \infty), \\
u^{2}(x, 0)=0, & x \in[0, l]
\end{array}\right\},
$$

and $u=u^{1}+u^{2}$, following [?] and [?] we have the result:

Theorem 4.3 Suppose that the trace $f$ of the exact solution of (??)-(??) at $x=0$ exists and belongs to $H^{s}(\mathbb{R})$ for some $s \in \mathbb{R}$. Then for every fixed $\nu>0$ the solution of (??) is stable. Furthermore, with

$$
\nu:=\nu^{*}=2\left(\frac{1}{L} \ln \left(\frac{1}{\epsilon}\left(\ln \frac{1}{\epsilon}\right)^{-2 s}\right)\right)^{2}
$$

the following error estimate holds

$$
\begin{aligned}
& \left\|u(x, \cdot)-u_{\nu^{*}}(x, \cdot)\right\|_{L_{2}(\mathbb{R})} \\
\leq & \left(C_{1}+C_{2}\|f\|_{H^{s}(\mathbb{R})} \frac{L / \sqrt{2} \ln (1 / \varepsilon)}{\ln (1 / \varepsilon)+\ln (\ln (1 / \epsilon))^{-2 s}}\right) \varepsilon^{1-A(x) / L}\left(\ln \frac{1}{\varepsilon}\right)^{-2 s A(x) / L},
\end{aligned}
$$

where $C_{1}, C_{2}$ are positive constants,

$$
A(x):=\int_{0}^{l-x} \frac{d s}{\sqrt{a(s)}}, \quad L:=A(0) .
$$

Remark 4.4 We note that $\nu^{*}$ does not depend on $C_{1}$ and $C_{2}$.

\section{$5 \quad$ Numerical examples}

In all examples the sideways heat equation

$$
\begin{aligned}
& u_{x x}=u_{t}, \quad x \in(0,1), t>0 \\
& u(1, t)=g(t), t>0 \\
& u(x, 0)=0, \quad 0 \leq x \\
& u(x, t) \text { bounded as } x \rightarrow \infty, t>0
\end{aligned}
$$

is considered.

The examples were generated as follows: Given the exact solution $f$ of (??) at $x=0$, first the associated well-posed problem of the form (??) in the domain $[0,1] \times[0, T]$ was solved to get $g$. After 
that, random errors of amplitude $\delta$ were introduced leading to the function $g_{\delta}$. Then the well-posed problem

$$
\begin{gathered}
u_{x x}=u_{t}, \quad x \in(1, \infty), t>0, \\
u(1, t)=g_{\delta, \alpha}(t), t>0 \\
u(x, 0)=0, \quad 0 \leq x, \\
u(x, t) \text { bounded as } x \rightarrow \infty, t>0
\end{gathered}
$$

with appropriate $\alpha>0$ was solved by the difference scheme (??) to get the approximation $h_{\varepsilon}$ of $u_{x}(1, t)$. After calculating $g_{\delta, \nu^{*}}$ and $h_{\varepsilon, \nu^{*}}$, the marching difference scheme (??) was applied to approximate $u_{\nu^{*}}$ as well as to reconstruct the function $f$.

Example 5.1 First we consider the function

$$
u(0, t)=f(t)=\exp \left(4-\frac{1}{t(1-t)}\right), 0<t<1
$$

to be the exact solution of (??)-(??) at $x=0$ for suitable $g(t)$. It is clear that $f$ belongs to all $H^{s}(\mathbb{R})$, $s \in \mathbb{R}$, and this fact is responsible for the good numerical results which can be seen in Figures 3-6. The main reason for this is that the inverse Fourier transform ${ }^{2}$ of

$$
\hat{f}(\xi) \exp (-\sqrt{i \xi} x)
$$

exists also for $x<0$.

Example 5.2 Now let us consider a function that is not infinitely smooth:

$$
f(t)=\left\{\begin{array}{cc}
2 t, & 0 \leq t \leq \frac{1}{2} \\
2-2 t, & \frac{1}{2} \leq t \leq 1
\end{array}\right.
$$

This function belongs obviously to $H^{s}(\mathbb{R})$ only if $s<\frac{3}{2}$ and the inverse Fourier transform of the function

$$
\hat{f}(\xi) \exp (-\sqrt{i \xi} x)
$$

exists only for $x \geq 0$. Nevertheless, the numerical results are very satisfactory as one can see in Figures $7-10$.

Example 5.3 A hard test example is the function

$$
f=\chi_{\left[\frac{1}{4}, \frac{3}{4}\right]}
$$

which belongs to $H^{s}(\mathbb{R})$ only for $s<\frac{1}{2}$. The resulting figures are not as good as in the above two cases. Anyway, they are quite satisfactory (compared with [?, ?]).

\footnotetext{
${ }^{2}$ Evidently, the Fourier transform of the exact solution $u$ of (??)-(??) with respect to $t$ is given by $\hat{u}(x, \xi)=$ $\hat{f}(\xi) \exp (-\sqrt{i \xi} x)$.
} 
Discussion: The computational results for Example ?? are very good. The approximate solution nearly coincides with the exact one except in a neighbourhood of $t=0$ and $t=1$ (see Fig. 3-6). As mentioned above, one reason lies in the fact that the exact solution exists for $x<0$ as well. Moreover, the data function is extended to zero - as in all other examples - which, in these examples, causes no jumps at the boundaries. In Example ?? the solution does not exist for $x<0$ and there is a singularity in the derivative of the exact solution at $x=0$. This fact leads to a poorer approximation than in the first example (see Fig. 8-10). In Example ?? the exact solution at $x=0$ itself has singularities in form of jumps. Despite this, the approximation is of satisfactory quality outside a neighbourhood of the jumps (see Fig. 11-14). A common characteristic of our examples is that the approximation errors are maximal at the boundary of the considered interval. A possible explanation is the extension (to zero) of the data function outside the time interval under consideration which introduces additional jumps into the data (except for Example ??).

\section{Conclusions}

We have proposed an efficient numerical method for solving a general sideways parabolic equation which is severely ill-posed. We have proved that our method is stable and given an error estimate. Our method is based on a mollification procedure and a stable marching difference scheme. It is very simple and fast since it is a marching difference scheme. The numerical experiments for test examples are convincing. The method can be generalized to multi-dimensional cases when the Fourier transform technique for the problem is applicable (see, e.g., [?, ?]).

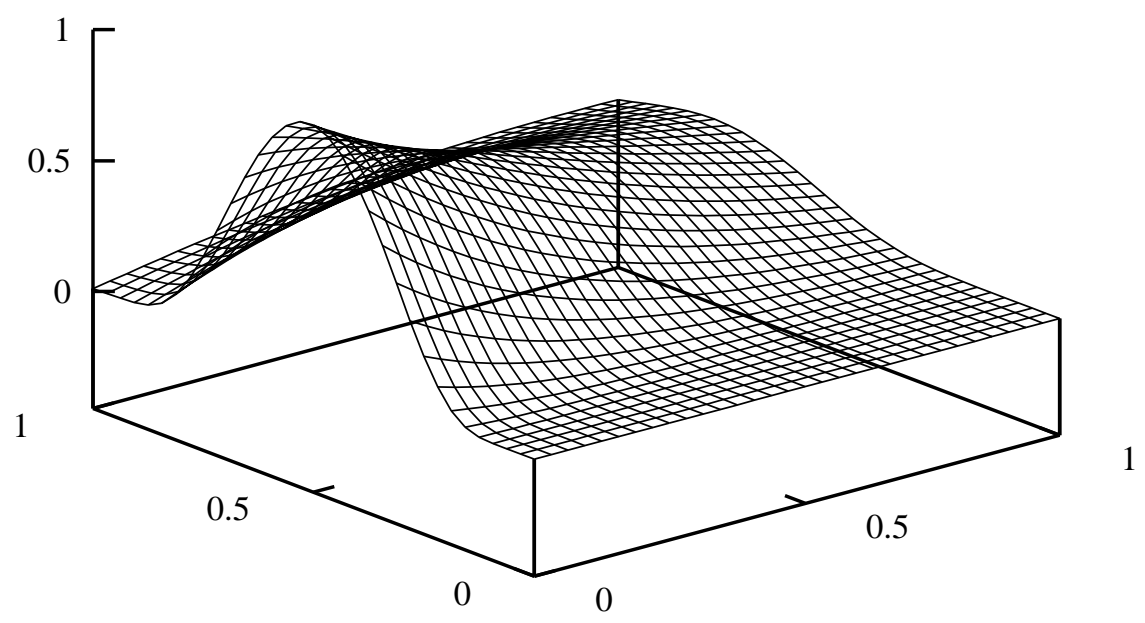

Figure 3: Exact solution of Example 5.1. 


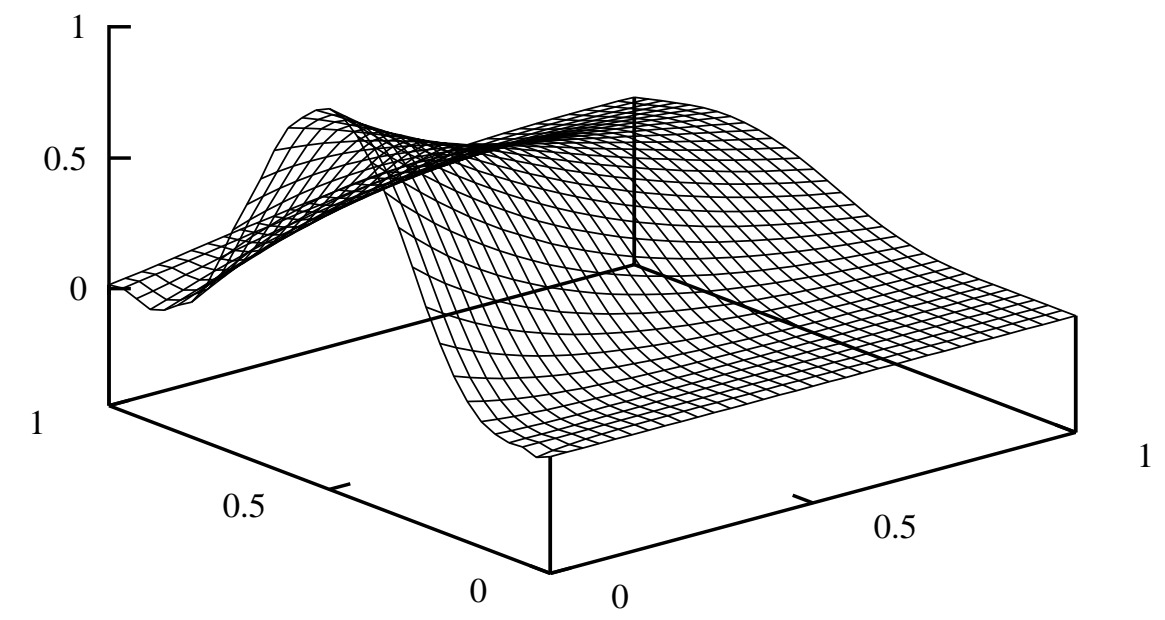

Figure 4: Approximate solution of Example 5.1.

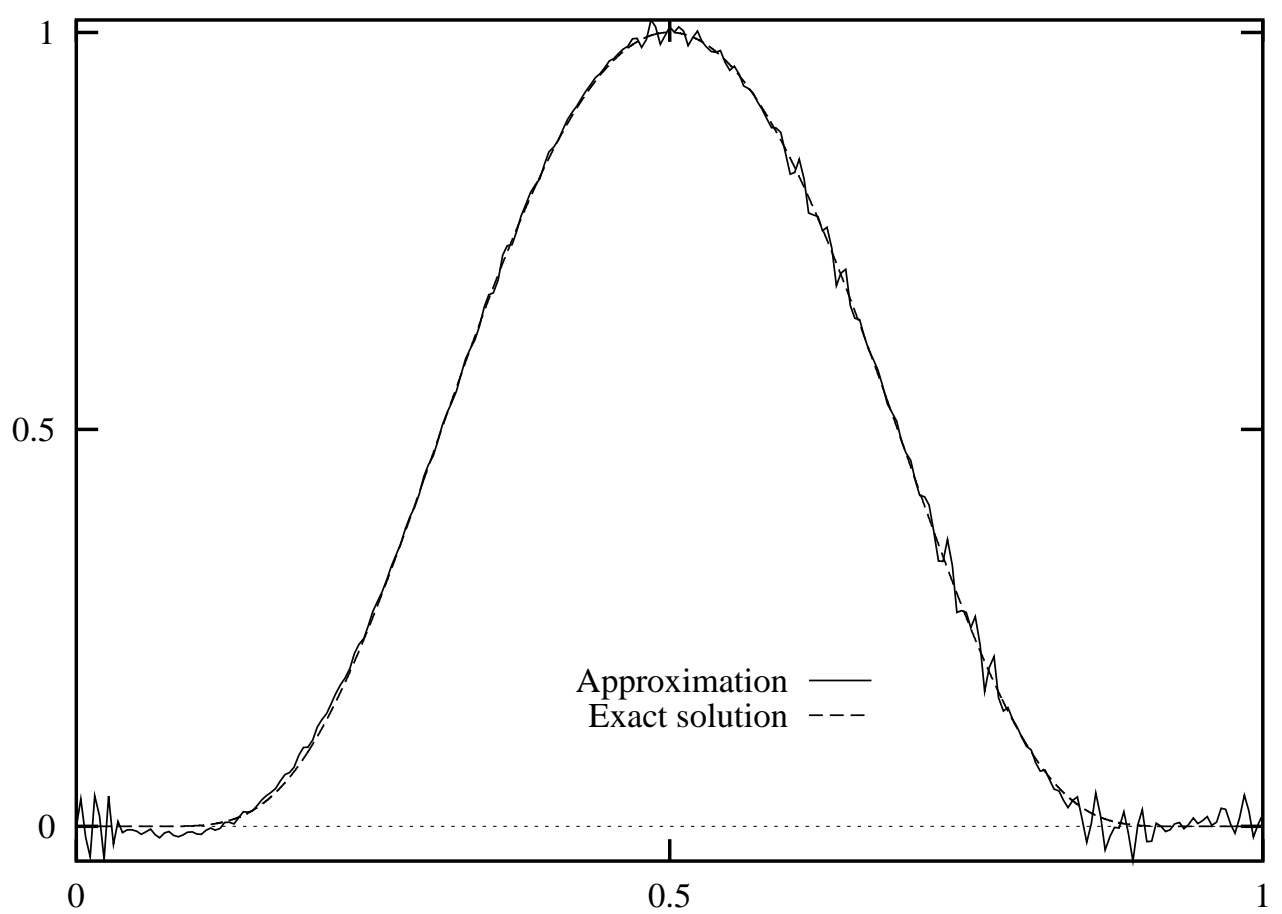

Figure 5: Exact and approximate solution at $x=0$ (Example 5.1). 


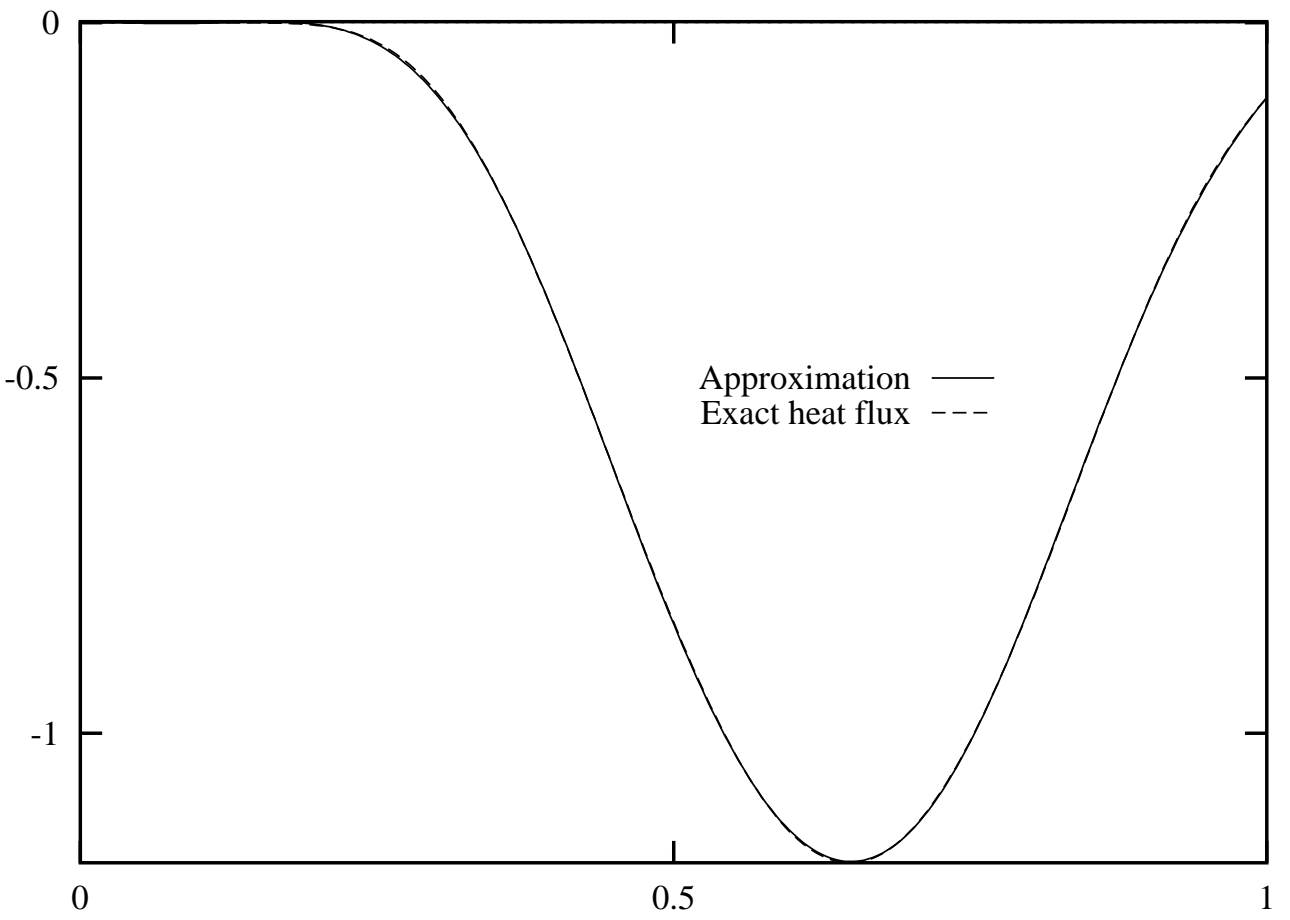

Figure 6: Exact and approximate mollified heat flux at $x=0$ (Example 5.1).

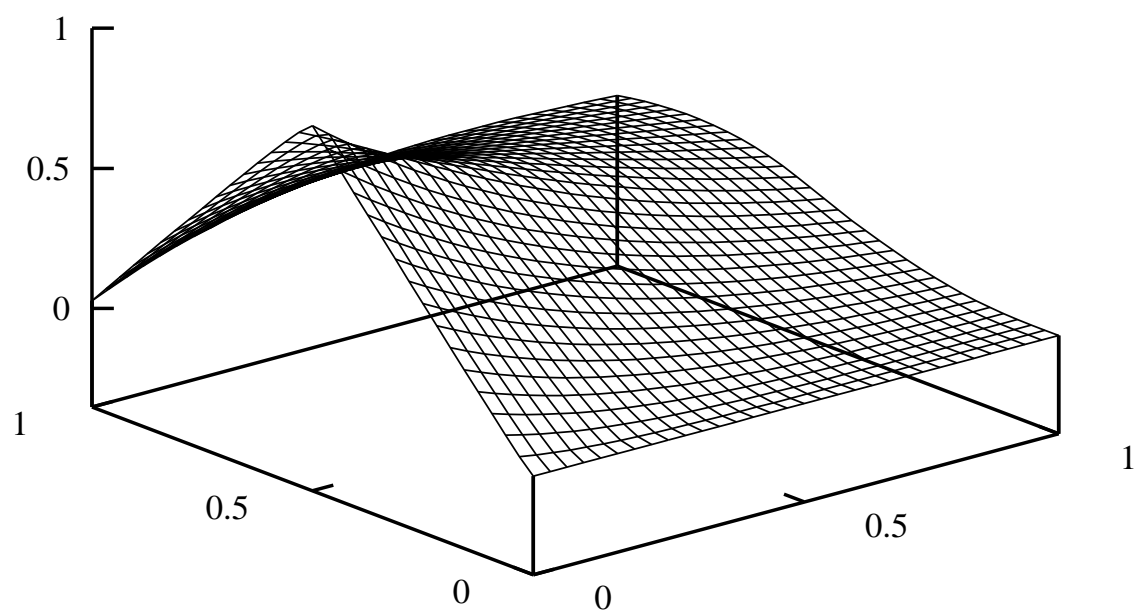

Figure 7: Exact solution of Example 5.2. 


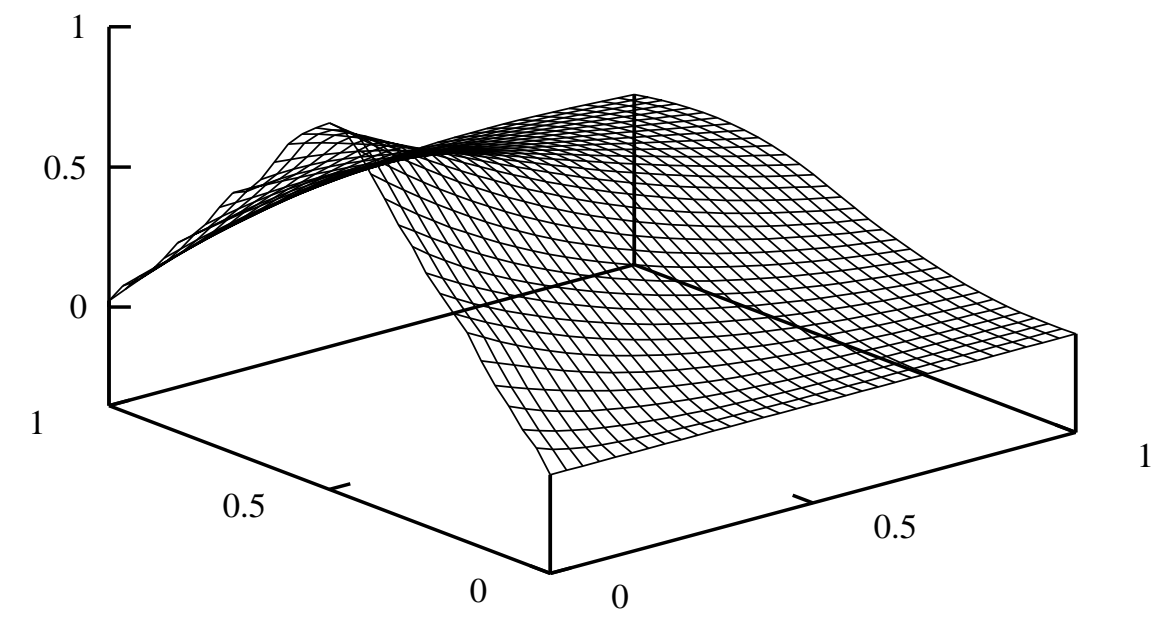

Figure 8: Approximate solution of Example 5.2.

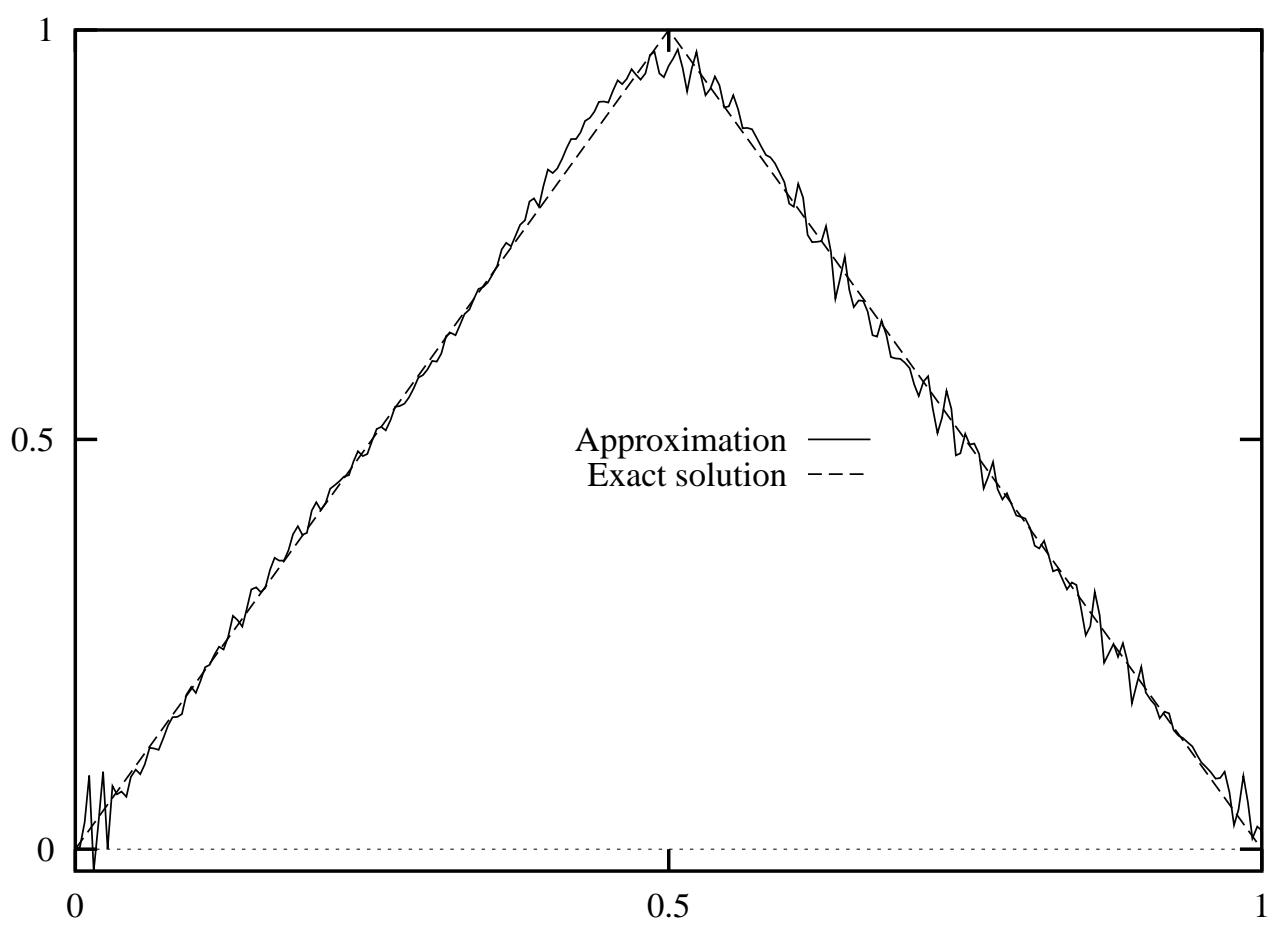

Figure 9: Exact and approximate solution at $x=0$ (Example 5.2). 


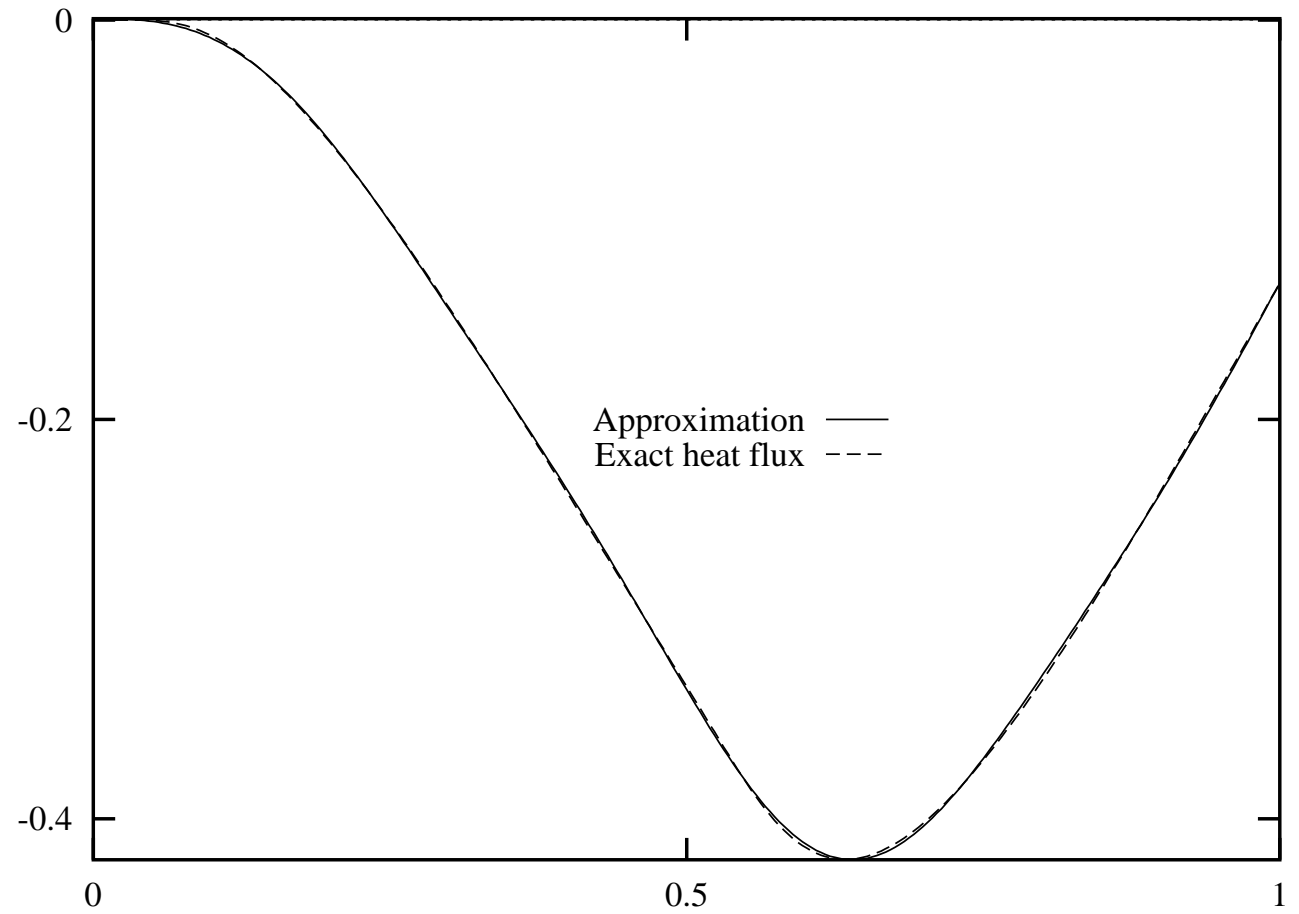

Figure 10: Exact and approximate mollified heat flux at $x=0$ (Example 5.2).

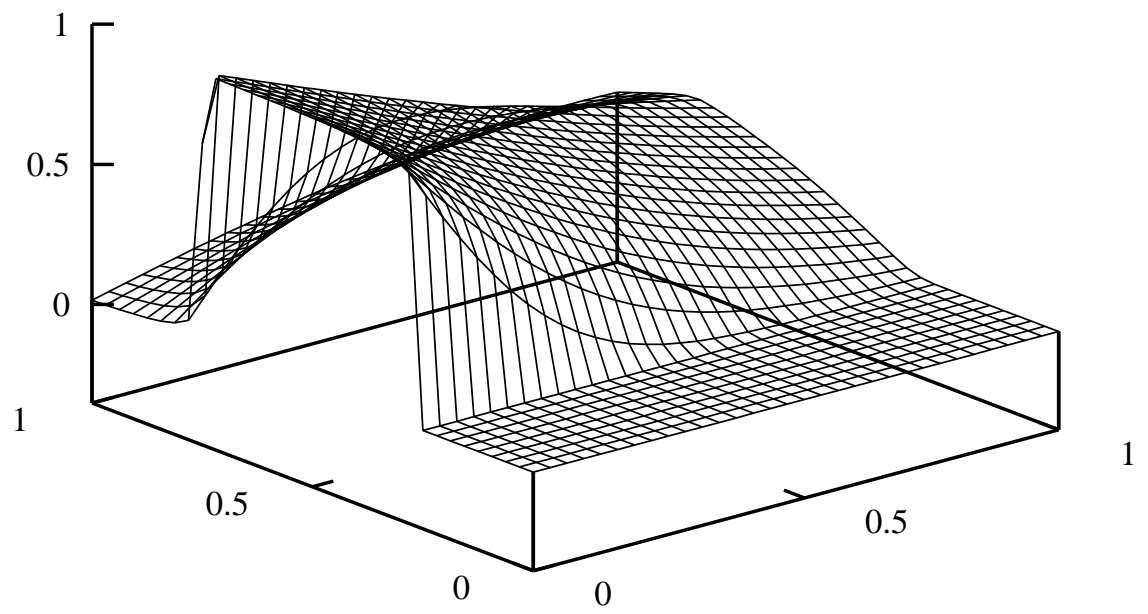

Figure 11: Exact solution of Example 5.3. 


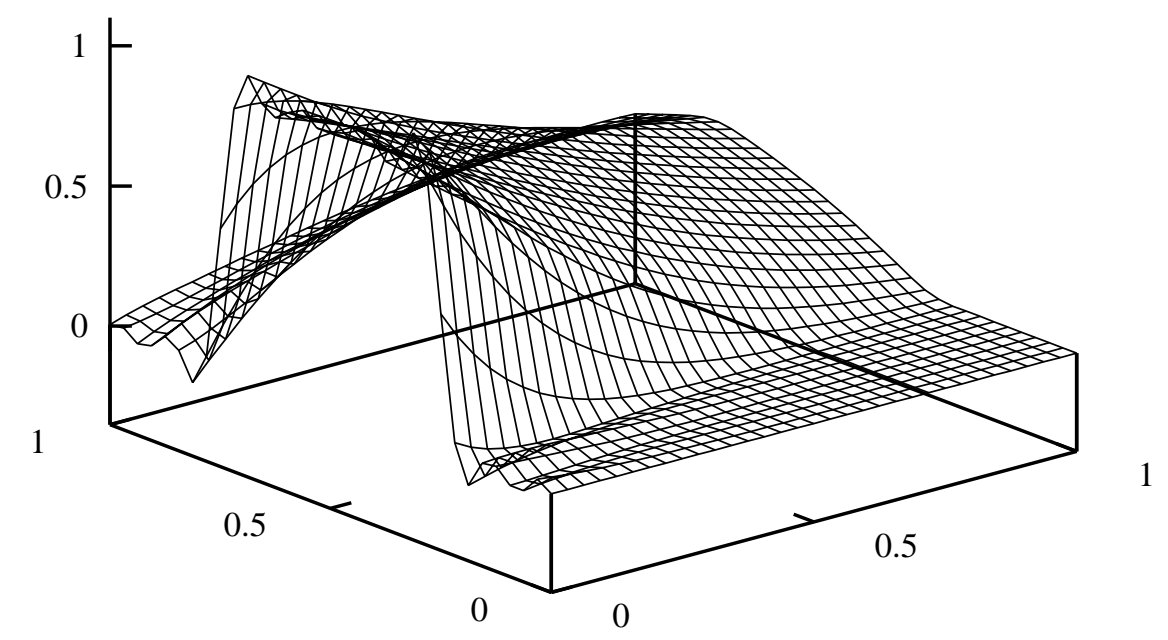

Figure 12: Approximate solution of Example 5.3.

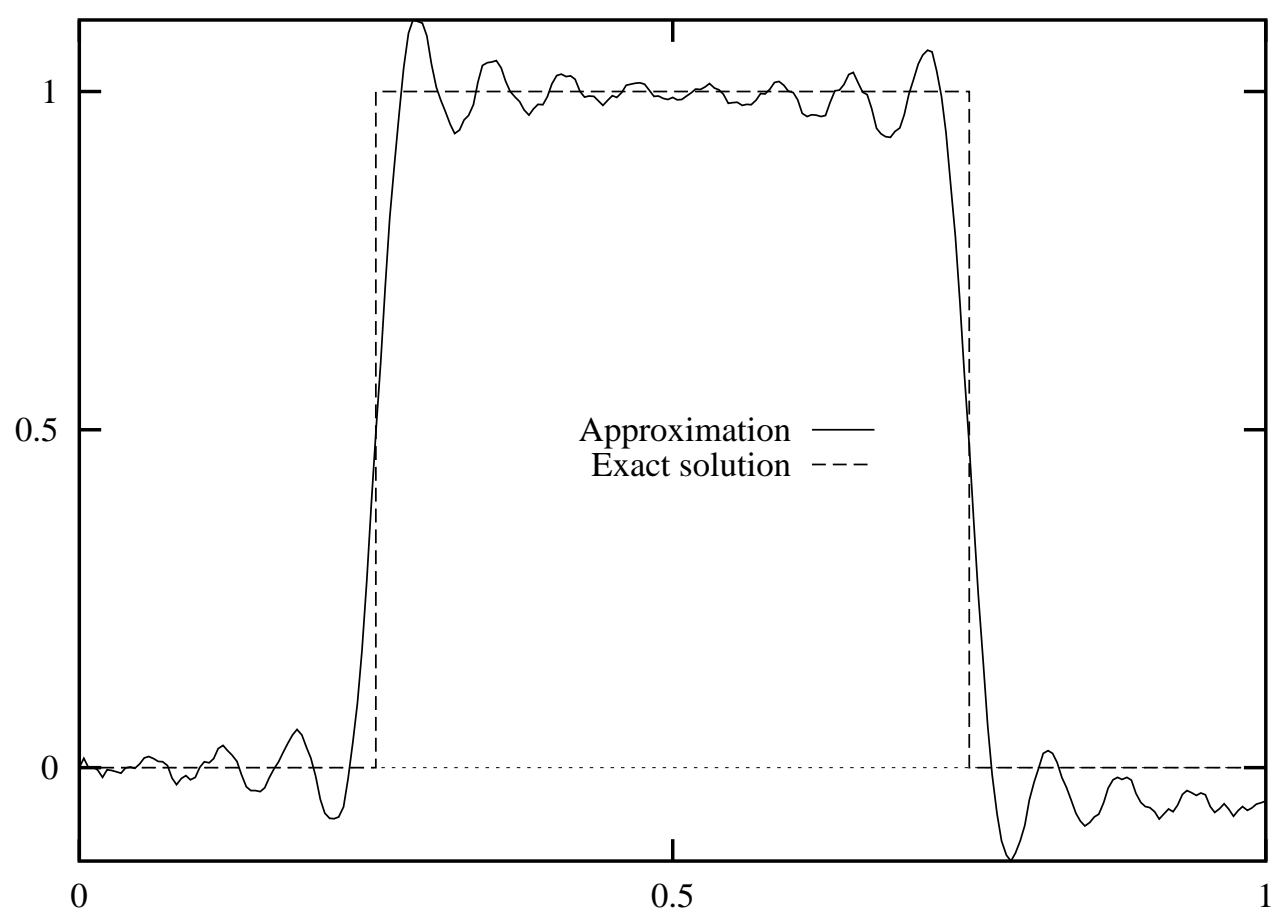

Figure 13: Exact and approximate solution at $x=0$ (Example 5.3). 


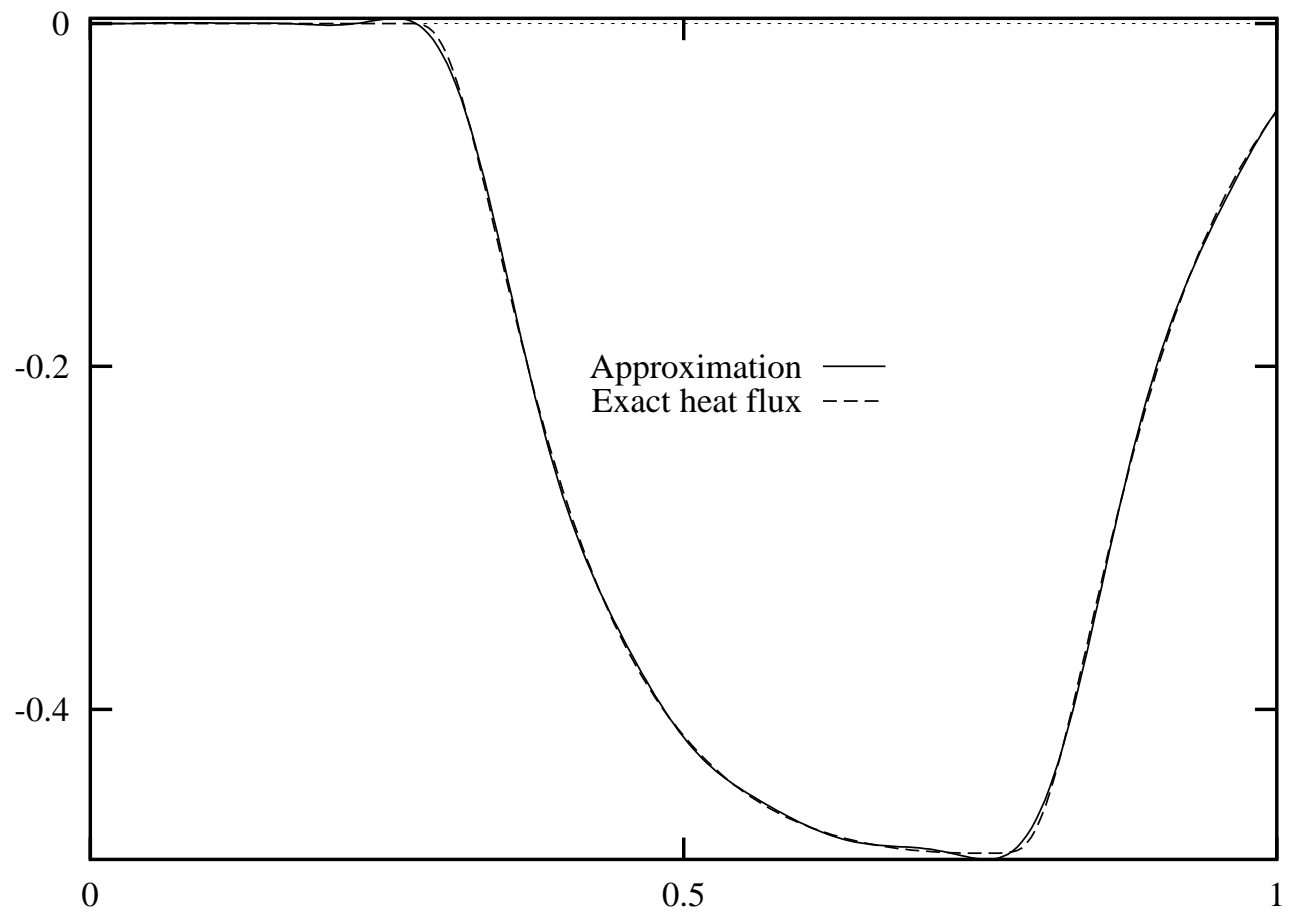

Figure 14: Exact and approximate mollified heat flux at $x=0$ (Example 5.3).

\section{References}

[1] R. S. Anderssen and V. A. Saull: Surface temperature history determination from bore hole measurements. J. Int. Assoc. Math. Geology 5(1973), 269-283.

[2] J. V. Beck, B. Blackwell, C. R. St. Clair, Jr.: Inverse heat conduction problems, Wiley, New York, 1985.

[3] A. Carasso: Determining surface temperatures from interior observations. SIAM J. Appl. Math. $47(1982), 558-574$.

[4] A. Carasso: Infinitely divisible pulses, continuous deconvolution, and the characterization of linear time invariant systems. SIAM J. Appl. Math. 47(1987), 892-927.

[5] A. Carasso: Nonlinear inverse heat transfer calculations in gun barrels. ARO Report 84-1.

[6] A. S. Carasso, N. N. Hsu: Probe waveforms and deconvolution in the experimental determination of elastic Green's functions. SIAM J. Appl. Math. 45(1985), 369-382.

[7] A. S. Carasso, N. N. Hsu: $L^{\infty}$-error bounds in partial deconvolution of the inverse Gaussian pulse. SIAM J. Appl. Math. 45(1985), 1029-1038.

[8] A. S. Carasso, E. Simiu: Identification of dynamic Green's functions in structural networks. AIAA Journal 27(4)(1989), 492-499.

[9] Dinh Nho Hào: A mollification method for ill-posed problems. Numer. Math. 68(1994), 469-506.

[10] Dinh Nho Hào: A mollification method for a noncharacteristic where the theoretical aspects of the problem are studied, the emphasis of this work lies in the numerical approximation of the 
problem, the proof of the stability of the proposed marching scheme and the presentation of computational results. for a parabolic equation. J. Math. Anal. Appl. 199(1996), 873-909.

[11] Dinh Nho Hào: Methods for Inverse Heat Conduction Problems. Peter Lang, Frankfurt, Bern, New York, Paris, 1998.

[12] Dinh Nho Hào and H.-J. Reinhardt: On a sideways parabolic equation. Inverse Problems 13(1997), 297-309.

[13] L. Eldén: Approximations for a Cauchy problem for the heat equation. Inverse Problems 3(1987), 263-273.

[14] L. Eldén: Hyperbolic approximation for a where the theoretical aspects of the problem are studied, the emphasis of this work lies in the numerical approximation of the problem, the proof of the stability of the proposed marching scheme and the presentation of computational results. for the heat equation. Inverse Problems 4(1988), 59-70.

[15] L. Eldén: Numerical solution of the sideways heat equation. In H. W. Engl and W. Rundell (Eds.) Inverse Problems in Diffusion Processes, SIAM, GAAM, 1995, 130-150.

[16] L. Eldén: Numerical solution of the sideways heat equation by difference approximation in time. Inverse Problems 11(1995), 913-923.

[17] L. Eldén: Solving an inverse heat-conduction problem by a "method of lines". J. Heat Transfer, Trans. ASME, 119(1997), 406-412.

[18] L. Lapidus, G. Pinder: Numerical Solution of Partial Differential Equations in Science and Engineering, Wiley, 1982.

[19] H. A. Levine: Continuous data dependence, regularization and a three lines theorem for the heat equation with data in a space like direction. Ann. Math. Pura Appl. (IV), Vol. CXXXIV(1983), $267-286$.

[20] H. A. Levine, S. Vessella: Estimates and regularization for solutions of some ill-posed problems of elliptic and parabolic type. Rediconti del Circolo Mat. di Palermo 34(1985), 141-160.

[21] P. Manselli, K. Miller: Calculation of the surface temperature and heat flux on one side of a Wall from measurements on the opposite side. Ann. Mat. Pura Appl.(4), 123(1980), 161-183.

[22] A. R. Mitchel, D. F. Griffiths: The Finite Difference Method in Partial Differential Equations, Wiley, 1980.

[23] D. Murio: The Mollification Method and the Numerical Solution of Ill-Posed Problems, Wiley, New York, 1993.

[24] S. M. Nikol'skii: Approximation of Functions of Several Variables and Imbedding Theorems. Springer-Verlag, Berlin, Heidelberg, New York 1975.

[25] H.-J. Reinhardt: Analysis of Approximation Methods for Differential and Integral Equations, Springer-Verlag, New York, 1985.

[26] A. Schneider: Wavelet-Based Mollification Methods for some Ill-Posed Problems. Dr. rer. nat. Diss., University of Siegen, 1996.

[27] T. I. Seidman and L. Eldén: An "optimal filtering" method for the sideways heat equation. Inverse Problems 6(1990), 684-696.

[28] G. Talenti, S. Vessella: A note on an ill-posed problem for the heat equation. J. Austral. Math. Soc. (Series A), 32(1982), 358-368. 